\title{
Adhesion-force micro-scale study of desiccating granular material
}

\author{
TOMASZ HUECKEL*, BOLESLAW MIELNICZUK† $† \S$ and MOULAY SAID EL YOUSSOUFI $\$$
}

\begin{abstract}
Experiments on five-, four-, three- and two-wet-hydrophilic-grain clusters were performed to investigate evolution of adhesion of granular media during drying on the micro-scale. The experiments show that the adhesion-force of a cluster initially grows at most to three times the original value before decreasing to zero by the end of evaporation. The adhesion-force is composed of capillary pressure force acting over the liquid/solid contact surface area, and surface tension forces acting over the three-phase contact perimeter length. This is in contrast with most macro-scale phenomenological models, in which the only desaturation process variables affecting strength are suction and saturation. Both the contact surface area and contact perimeter length are reduced to zero upon complete liquid evaporation. The morphology of an evaporating water body evolves through slow flow controlled by evaporation rate, interrupted by various modes of fast air entry, which are non-equilibrium jumps of liquid/gas interfaces (Haines jumps). The instabilities involve large adhesion force discontinuities and substantial water mass reconfiguration with water flow in an extremely short time, which makes the process transient. The reconfigurations can reduce the original multi-grain water clusters to four-, three- and two-grain clusters by way of three different instability modes: of thin-sheet instability, or meniscus snap-through instability, depending on the sign of the Gauss curvature of the liquid surface, or finally, for two-grain bridges only, a liquid wire pinch-off. For larger meso-scale assemblies, however, the global adhesionforce evolution is little affected by the jumps. The air entries are potential sites for drying cracks. The (approximately) calculated capillary pressure for two- and three-grain clusters, in no cases is seen to reach high values, predicted from water retention curves.
\end{abstract}

KEYWORDS: dewatering; laboratory tests; partial saturation; particle-scale behaviour; pore pressures; suction

\begin{abstract}
INTRODUCTION
There is a substantial difference of approach to the strength of desaturating, granular materials at the level of the microscale (grain-size) and macro-scale (continuum) medium. At the micro-scale, the strength of an externally unloaded cluster of grains is expressed through attraction between grains by way of self-equilibrated adhesion forces (or intergranular forces) starting with Young's (1805) seminal work entitled 'An essay on the cohesion of fluids', whereas at the macro-scale it is expressed by way of a property of cohesion, or shear, or tensile, or compressive, or preconsolidation strength (often formulated through a yield stress locus size).

Adhesion produced at the micro-scale between individual or clustered particles is a resultant force (positive, when attractive) developing across any cross-section of a particle cluster as a result of several phenomena: Laplace pressure in the liquid trapped between the solids and an interface with gas, acting at the liquid/solid interface; surface tension along the triple line interface between gas, liquid and solid, introduced by Fisher (1926); and finally solid-solid attraction (Fisher \& Israelachvili, 1981; see also Chang \& Hicher, 2009). The solid-solid forces most commonly are
\end{abstract}

Manuscript received 8 November 2018; revised manuscript accepted 4 September 2019. Published online ahead of print 11 October 2019. Discussion on this paper closes on 1 April 2021, for further details see $p$. ii.

* Duke University, Durham, USA (Orcid:0000-0001-8293-5472).

$\dagger$ Duke University, Durham, USA (Orcid:0000-0002-4933-5077).

† LMGC UMR 5508, CNRS-Université de Montpellier, France.

$\S$ MIST Laboratory, IRSN-CNRS-Université de Montpellier, France. negligible at solid gaps larger than tens of nanometres (Israelachvili, 1992).

Cohesion at the macro-scale is a strength (attribute of failure) property of a granular continuum, defining a critical shear stress at a null (mean) normal stress (Coulomb criterion), or independent of the mean stress (Tresca or Huber-von Mises criterion); see, for example, Mitchell (1976).

The link between the adhesion and cohesion is intuitive rather than rigorous. Adhesion can be viewed as a selfequilibrated residual force system, meaning, with no external load needed to keep a moist cluster together. Cohesion is defined as the shear stress resulting from an external load, possibly added to the residual stress, which brings the system to failure at no (mean) normal stress (at a potential rapture surface). A possible link between tensile strength and adhesion is often suggested by way of a maximum resultant interparticle force across a representative cross-sectional area (Rumpf, 1962; Cheng, 1968; Schubert, 1975).

One of the intriguing macro-scale properties of an unsaturated granular medium is that, upon a change in saturation, its cohesion considerably departs from the values at a dry state, or those at a completely saturated state. Any such departure is of central interest to geomechanics and geotechnics, as it controls the shear strength of the material, and thus the material conditions of failure. Other strength descriptors, such as compressive unsaturated strength (Soulié et al., 2006; Mokni et al., 2014) and tensile strength in a particular effective stress convention (Lu et al., 2009) also show a strong dependence on the saturation degree.

In geotechnical applications, cohesion of granular soils in unsaturated conditions, or 'apparent cohesion', is postulated to consist of two components, namely: shearing resistance at zero normal external stress, attributed mainly 
to intergranular friction and van der Waals forces, and capillary cohesion (see e.g. Taylor, 1948; Fredlund et al., 1978; Fisher \& Israelachvili, 1981; Fredlund \& Rahardjo, 1993; Lu \& Likos, 2004; Soulié et al., 2006; Richefeu et al., 2007). The capillary cohesion is postulated to be a function of macro-scale matric suction, widely reported to be very high at low saturation (Taylor, 1948; McQueen \& Miller, 1974; Romero et al., 2011; Mokni et al., 2014), reaching values of the order of $0 \cdot 8-600 \mathrm{MPa}$. There are conflicting data suggesting the capillary cohesion to be a decreasing function of water content, $w$, for $w<13 \%$ (Soulié et al., 2006; Lu et al., 2007).

In continuum constitutive models of unsaturated soil mechanics fashioned after Cam Clay plasticity theory, cohesion is related functionally to an apparent preconsolidation pressure variable, postulated as a power function of a suction-dependent plastic compressibility (e.g. Alonso et al., 1990; Gens \& Alonso, 1992; Wheeler \& Sivakumar, 1995; Sun et al., 2007). Alternatively, macro-scale cohesion is considered as a variable through independent phenomenological mechanisms controlled by two variables: suction and saturation (Laloui \& Nuth, 2009), or suction and effective degree of saturation (of macropores), see Alonso et al. (2010) or additionally, an interfacial surface area (Likos, 2014).

Suction evolution, as classically represented in geotechnical and agricultural engineering through a soil water characteristic curve (SWCC), exhibits values increasing up to about $2 \mathrm{MPa}$, at water contents of $9-10 \%$ (Fredlund \& Rahardjo, 1993; Mitchell \& Soga, 2005). It is not unusual to see up to $800 \mathrm{MPa}$ of suction needed to reach water contents below 5\% in SWCC (McQueen \& Miller, 1974; Likos, 2000; Lu \& Likos, 2004). Exceedingly high values of suction in SWCC raised objections for a number of years, for various reasons, one of them being lack of any evidence of grain crushing that would result from the corresponding huge effective stress at zero total stress. Gens (2010) cautions against a strict interpretation of suction in soil mass as a negative resultant bulk water pressure. Thus a question arises: what could be other possible phenomena that may interfere with the matric suction effects?

This study aims at understanding how adhesion, cohesion and strength, in general, evolve in a granular medium during desaturation by way of desiccation. The approach is through studying a micro-scale behaviour of capillary water in small clusters transitioning from $(a)$ capillary to $(b)$ funicular and finally to $(c)$ pendular configurations during drying (see Newitt \& Conway-Jones, 1958; Schubert, 1984). The described approach to the unsaturated soil strength is based on the total intergranular adhesion-force, with its two components, the resultant of the Laplace (capillary) pressure and the surface tension force (Fisher, 1926; Hotta et al., 1974; Willett et al., 2000). The pressure forces act over the contact surface area, while surface tension forces act along the contact perimeter, both of which become highly variable during the process of evaporation. Both are also vanishing with the decreasing volume of the liquid phase. The intergranular force evolution associated with evaporation is measured, while the Laplace pressure and surface tension forces are assessed by way of Laplace law. The models employed are all two-dimensional (2D), composed of spherical smooth glass grains, immobilised at a predetermined distance from one another. The space between the grains is filled with deionised, chemically inert water of controlled initial volume. In particular, the differences between evolution patterns occurring at different separations for various five-, four-, three- and two-grain particle set-ups are examined. Consequences to the understanding of unsaturated granular medium mechanics are highlighted.

\section{MICRO-SCALE EXPERIMENTS}

The general context of a desaturating medium

In macro-scale experiments, to relate cohesion, or strength in general, to suction, it is customary to first bring an originally unloaded sample to a desired level of saturation, and then at a constant saturation, load the material until yielding (see e.g. Delage, 2013). At micro-scale, however, the attractive adhesion force has its maximum at a close contact of the grains (almost, but not necessarily, at zero separation) and decreases during an increasing separation, lowering the strength of adhesion, as seen in experiments with evaporation and extension of two-grain systems (Mielniczuk et al., 2014a). Thus, in a process consisting of an initial desaturation, followed by an extension, the extension part results in the interparticle bond softening. Hence, the highest adhesion (tensile) force develops at zero extension strain. In other terms, its maximum cohesive force, or a cohesive force at the onset of failure, is equal to the initial adhesion. This is equivalent to the strain-softening behaviour of the cohesion upon extension. Such results have been obtained many times before (see e.g. Mason \& Clark, 1965) and a classical theoretical formulation for the test has been available for a long time (see e.g. Lian et al., 1993). An analogous argument has been made for three-grain bridges by Murase et al. (2004, 2008).

\section{MATERIALS AND METHODS}

All the experiments were performed in the apparatus presented in Fig. 1, as described in detail by Gras et al. (2013) and Mielniczuk et al. (2014a). The device is designed to measure the intergranular force across and acquire images of the capillary bridge between two sets of grains: a lower grain set sitting on a scale, and an upper set hanging from a fixed support. A prescribed amount of distilled and deionised water, with surface tension coefficient $\gamma=0.072 \mathrm{~N} / \mathrm{m}$, is injected between the grain sets. For a given water volume and given grain separations the resulting liquid bridges were exhibiting high repeatability, and considered to be those of initial equilibrium. The change of the 'apparent weight reading' for the lower grain set at the instant of the water bridge formation is considered to be equal to the initial intergranular force in the bridge. Further changes of the apparent weight are attributed to the intergranular force changes due to evaporation. The experimental procedures are described by Mielniczuk et al. (2014a). A digital camera continuously records the whole process. To image the moment of rupture, a high-speed camera (Vision Research Phantom v12) is used, with the rate of more than 40000 frames per second. The separation between the two sets is set using a micrometric screw. The tests are performed by enabling evaporation at a constant relative humidity in the test chamber at pre-defined constant separations.

It is customary for unsaturated media to present the evolution of variables as a function of degree of saturation. A similar representation will be used here, but referenced to a few-grain cluster, rather than to the medium. As the volume of pores is not defined in a unique way for a cluster, the reference volume of pores (constant) is assumed to be equal to the initial total volume of water. Hence, cluster saturation $S$ varies between 1 (for the initial volume of water) and 0 (no water left) during the bridge evaporation. Changes in cluster saturation include the loss of water evaporating in front of and behind the set-up, not observable in the images. Cluster saturation is by no means numerically equivalent to saturation of the medium. Indeed, it is worth noting, for comparison, that saturation of a regular monodisperse cubiccentred granular medium at the moment of creation of pendular (two-grain) bridges is about 9\% (Luikov, 1966), 


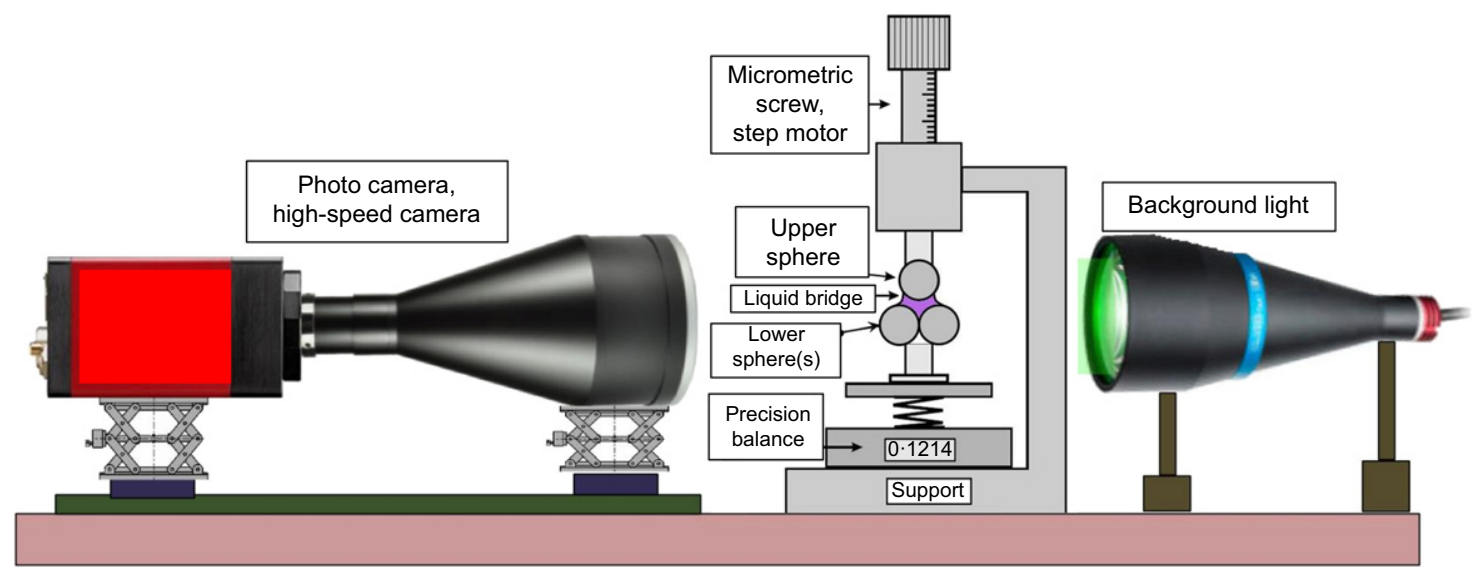

Fig. 1. Apparatus employed to measure total intergranular force for systems of two to eight wet grain clusters. The upper sphere(s) in the device are attached to the frame, while the bottom grains sit on the precision balance. The apparent weight read by the balance is the weight of the bottom spheres and water, less the adhesion force exerted by the water body on the bottom spheres (see Mielniczuk et al. (2014a) for details)

while cluster saturation of a pair of grains at that moment is $100 \%$.

There are several points that need to be kept in mind in qualifying these results.

(a) First, drying flux is generally deemed to be uniform at all external surfaces exposed to air. This assumption is questionable, as there is wide evidence to the contrary on the micro-scale, for both evaporation of sessile drops, and for drying bridges (Deegan et al., 1997; see also Yang et al., 2018).

(b) Second, it has been noted in a number of recent meso-scale tests that the pressure-saturation relationship of evaporating (or variable saturation) media (SWCC curve) substantially depends on the driving variable, and hence it is different for pressure-driven, and for saturation-driven tests (Toussaint et al., 2012; Armstrong \& Berg, 2013; Armstrong et al., 2015; Cueto-Felgueroso \& Juanes, 2016; Helland et al., 2017). The present authors' tests qualify as environment-controlled tests, as constant temperature and constant relative humidity are imposed in a test chamber.

(c) Third, the tested grain clusters do not suffer much from the effect of gravity, even clusters as large as eight grains, especially for a relatively small size of spheres $(3.5 \mathrm{~mm})$ and small separations between grains.

(d) The fourth and final point is that a single-grain arrangement does not necessarily yield a unique water morphology evolution, as the air entry onset mode and its subsequent morphology depend on the grain local separations, specifically on the ratio of separation to the grain radius. For specific arrangements in symmetric systems, initial air entry depends on a random distribution of small system imperfections.

\section{RESULTS}

The most important quantitative result of this work is the assessment of the intergranular force evolution during liquid bridge evaporation. It informs a major fraction of the modelling process of the mechanical behaviour of unsaturated granular assemblies. Intergranular force evolution is directly linked to transformation of a water body during evaporation, consisting of two well-articulated, alternating and drastically different kinematic patterns: a relatively slow (incremental) motion of the external air/water interface at a rate equal to that of evaporation, followed by a sudden acceleration with a substantial alteration of the interface localised to a span between two grains (instability), viewed as 'the air entry', resulting in a water mass rearrangement to a completely different configuration. Further evaporation may induce a repeated sequence of a slow motion of the interface, until a next unstable fast reconfiguration of the interface.

The cluster adhesion force or cluster intergranular force is measured for a series of five, four, three, or finally two grain clusters in different grain arrangements, mainly different separations, between a bottom row and a top row of grains. The common features of the adhesion force evolution are the force jumps corresponding to instabilities of the water body geometry and water mass rearrangement. The sign of the local Gaussian curvature (GC) of the external surface at a point of instability (negative and positive, marked, respectively, by $\mathrm{N}$ and $\mathrm{P}$ ) appears to control the type of instabilities that cause force jumps, shown in Fig. 2 for a three-grain cluster. The instabilities are either in the form of an ultra-fast growing circular hole in a thin film, which develops at a point of a positive GC (bowl mode), Figs 2(b) and 2(c), or in the form of a finger that undergoes fast snap-trough transformation at a saddle point to an alternative position of equilibrium, Fig. 2(d).

A separate issue is the 'air entry' for two-grain bridges, which is equivalent to a bridge rupture that also occurs over a very brief duration as yet another instability in the form of a water wire pinch-off. The precursors to rupture and its evolution have been discussed by Mielniczuk et al. (2015).

For the five-grain clusters the adhesion force measured for two set-ups (called in what follows loose and tight) and its evolution is presented in Fig. 3(a) (loose clusters), and in Fig. 3(b) (tight clusters). Distances between grains for both configurations are presented in Fig. 3(c) (loose clusters, with gaps between beads in the same row) and Fig. 3(d) (beads in row are in contact).

For both loose and tight clusters alike, the adhesion force value strongly depends on the separation $D$, set up as shown in Fig. 2(a): the smaller the separation, the higher in general, is the force.

In the case of the loose clusters (Fig. 3(a)), the intergranular force first increases with the decreasing cluster saturation, for all separations, except for the largest one, with the biggest increase (nearly triple) for the smallest separation $(0.1 \mathrm{~mm})$. The largest maximum force of $0.9 \mathrm{mN}$ is obtained for the smallest separation, at about $3 / 4$ of water evaporated, while the smallest maximum initial force $0.5 \mathrm{mN}$ is measured for the largest separation. Also, the smaller the separation, the later is the occurrence of the force maximum in the 


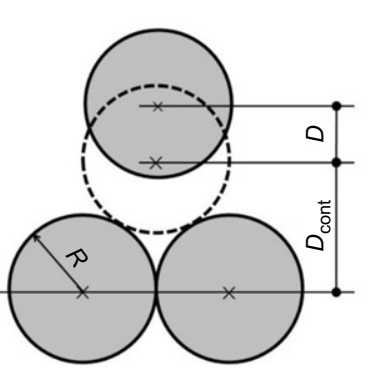

(a)

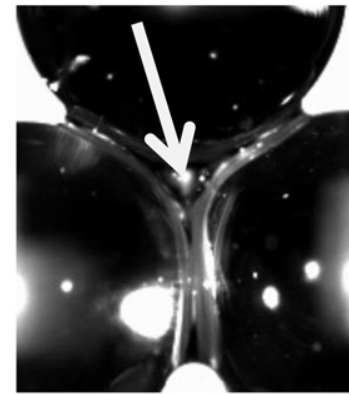

(b)

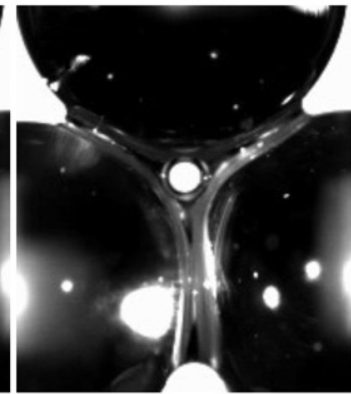

(c)

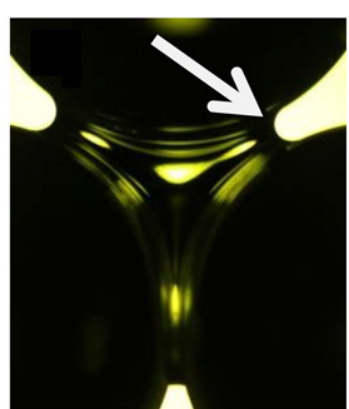

(d)

Fig. 2. (a) Definition of separation $D$ in multi-grain systems. Two typical instabilities in 2D systems of three grains: (b) and (c) tight clusters: $D / R=0 \cdot 025$, arrow indicates a point of minimum positive Gauss curvature; (c) thin-sheet instability for tight system, see text; (d) a loose system: $D / R=0 \cdot 175$, the arrow indicates a point of maximum absolute value negative Gauss curvature

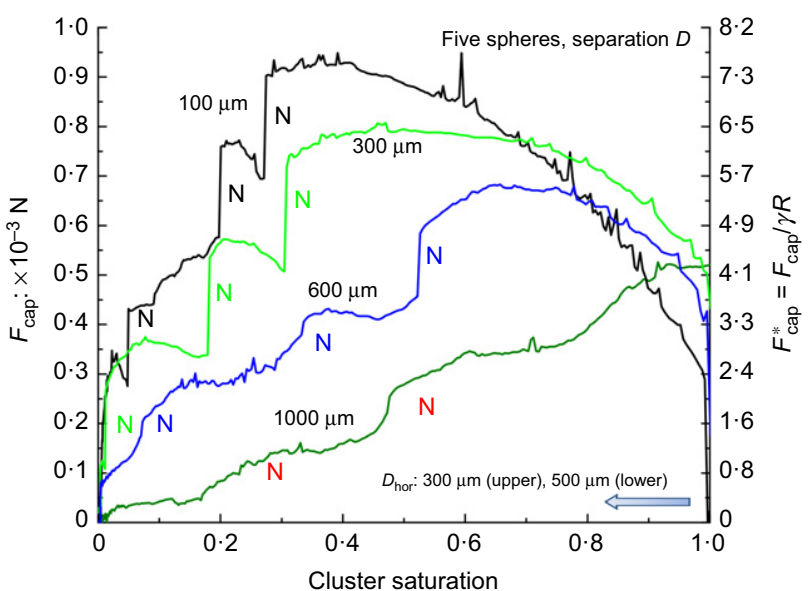

(a)

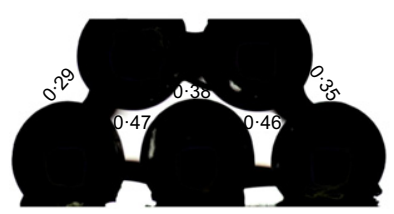

$D=0.1 \mathrm{~mm}, V_{0}=20 \mu \mathrm{l}$

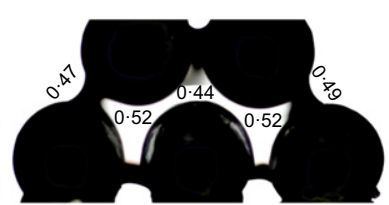

$D=0.3 \mathrm{~mm}, V_{0}=20 \mu \mathrm{l}$

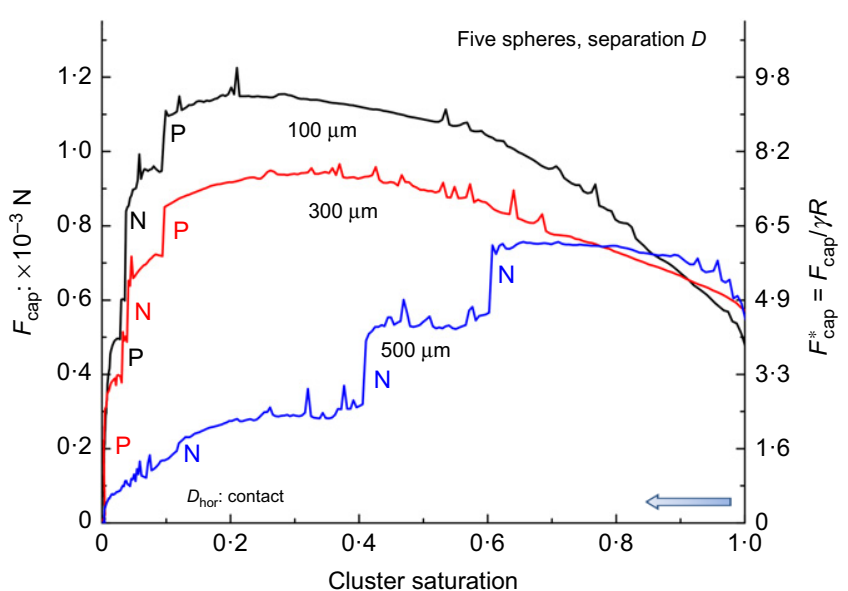

(b)

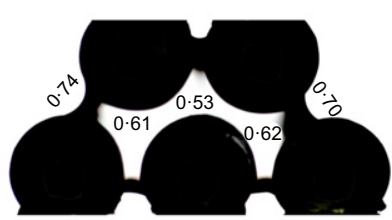

$D=0.6 \mathrm{~mm}, V_{0}=20 \mu \mathrm{l}$

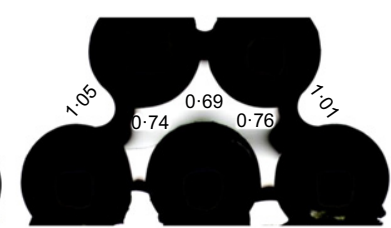

$D=1.0 \mathrm{~mm}, V_{0}=20 \mu \mathrm{l}$

(c)
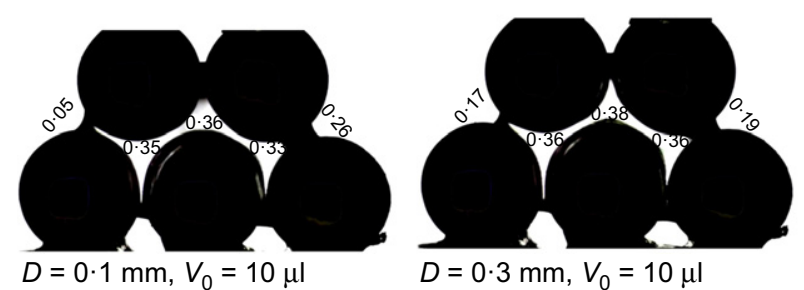

$D=0.3 \mathrm{~mm}, V_{0}=10 \mu \mathrm{l}$

(d)

Fig. 3. Evolution of total adhesion force, $F_{\text {cap }}$, during evaporation for five-grain (3.5 mm dia.) clusters (a, b). Parts (c) and (d) show the geometrical characteristics of cluster pore system: at the marked separations, $D$, between top and bottom row grains and separation between grains. (c) loose systems: non-zero horizontal gaps, $D_{\text {hor }}=300 \mathrm{~mm}$ (top) and $500 \mathrm{~mm}$ (bottom); side gaps, and central gaps (inscribed circle radius) as marked. (d) tight systems: zero horizontal gaps; side gaps, and central gaps (inscribed circle radius) as marked. The drying process from initial water volume, $V_{0}$, is represented in parts (a) and (b) from right to left, with cluster saturation varying between 1 and 0 , as in classical water retention curves and shown by the arrows. $N$, negative Gauss curvature instability; $P$, positive Gauss curvature instability

process. After the maxima, the intergranular force in all the cases decreases down to zero.

Most notably, total intergranular force displays strong discontinuities (reduction jumps) in correspondence to the instability events, shown in Fig. 2, of the order of $0.2 \mathrm{mN}$, which is about $20 \%$ of the maximum force value. These jumps are somehow attenuated for larger separations, becoming nearly smooth transitions. There is a slight recovery of the force, after the drop (best seen for $0.1 \mathrm{~mm}$ and $0.3 \mathrm{~mm})$, as the system reconfigures the masses of water displaced by the air entry, see again in Figs 2(c) and 2(d).

For the tight clusters, the intergranular forces are about $10 \%$ higher on average compared to the loose clusters and exhibit similar tendencies. The main difference is in the fact that both maxima and jumps occur slightly later in the process for tight systems. 
Notably, larger capillary systems (five grains, in the present case) evolve through a series of unstable events (jumps) to a number of smaller sub-systems (four-, three- and two-grain bridges. However, these smaller sub-systems exhibit high similarities in the behaviour of the intergranular forces.

The evolution of capillary force during evaporation of a four-, and a three-grain system, for different values of separation, is presented in Fig. 4. As the grain diameters are different, one has to use a normalised force scale for the quantitative comparisons. Such results were first obtained by Gras (2011).

For the cases presented, the trends in evolution are quite similar to five-grain assemblies. For smaller separations, the capillary force consistently initially increases, and then decreases to zero, whereas for larger distances it decreases right from the very beginning, with the instabilities and force jumps occurring at less than $1 / 4$ of water evaporated. When there is a force maximum, it occurs later in the process for the smaller separations. For a four-grain bridge at $0.6 \mathrm{~mm}$ separation, the instability mode is that of a finger at a saddle meniscus, with a very similar kinematics, whereas for tighter bridges for $0 \cdot 4,0 \cdot 3$ and $0.1 \mathrm{~mm}$ the first instability is at a bowl point with the thin-sheet instability. Notably, the second instability and the force jump occur at a very small volume of water left even for $0 \cdot 1 \mathrm{~mm}$.

For three-grain bridges, the force is initially either increasing or decreasing depending on the separations. For all separations, there is a substantial first jump of force, on average of $0.2 \times 10^{-3} \mathrm{~N}$ (between 50 and $30 \%$ of the original force), corresponding to the occurrence of the interface surface instability, independently of the instability mode. Phenomenologically, there is a correlation between the initial force-volume loss slope and the type of instability. For positive initial slopes, the initial instability is at the point of negative Gauss curvature, and vice versa, for initial negative slope, the initial instability takes place at the positive Gauss curvature point.

After the first instability, there is a slight recovery from the value of the force at the bottom of the jump, corresponding to a re-equilibration of the water masses. There are two possible post-instability configurations, with two or three two-grain (or pendular) bridges, depending on the type of instability (thin-sheet mode or finger/saddle mode). In the cases considered, one of these bridges is perpendicular to the direction of the measured intergranular force, and hence should not contribute to the value of the latter. These two-grain sub-sets in turn rupture in the ulterior process of evaporation, especially longer bridges, as explained below (see also Mielniczuk et al. (2014a, 2014b)). However, the force jumps related to these instabilities are clearly much smaller than the first instability jumps. Nevertheless, the moment, or the amount of water that has not evaporated at the point of the second instability, is crucial, as it determines the value of macroscopic residual saturation degree, and effectively the point of loss of cohesion. At the same time, the remaining amount of liquid water ends up forming a thin film layer around the particles, subject to an ulterior evaporation. An important observation to make is that in general the adhesion-force character for five-, four- and three-grain systems seems to be independent of whether the instability is of a negative or a positive Gauss curvature type. However, for tighter systems, the preferential instability type is that at a bowl-shaped point starting at a positive Gauss curvature. For loose systems the dominant instability type is at a saddleshaped location (with negative Gauss curvature).

The final issue is that of the evolution of intergranular force during evaporation of two-grain bridges. The results of testing of this phenomenon have been exhaustively reported by Mielniczuk et al. (2014a, 2015) and thus are only briefly summarised here for completeness. Fig. 5(a) reproduces the variation of the total intergranular force, against the cluster saturation. As in almost all of the cases before, the smaller the separation, the higher is the force. However, at the decreasing separations, the force increases by a modest amount, rather than exponentially, and the values do not reach more than $0.75 \times 10^{-3} \mathrm{~N}$. For the three smallest separations the force increases during evaporation not more than $20 \%$ of the initial value. After the maximum, the decrease is smooth and gentle, except for the smallest, $0.01 \mathrm{~mm}$ separation, where having lost about $95 \%$ of bridge water, the intergranular force rapidly drops from $0.5 \times 10^{-3} \mathrm{~N}$ to 0 , at practically the same volume. For the larger separations of $2 \cdot 0,1 \cdot 3$ and $0.7 \mathrm{~mm}$, hence for the most slender bridges, the bridges rupture, having lost $1 / 4$, or $1 / 2$ or $3 / 4$ of water volume, with the force reduced by a $1 / 4,1 / 2$ or $3 / 4$. As described earlier, the kinematics of the rupture through the water-wire instability occurs with the rate by far exceeding that of evaporation loss, hence it is believed to be mechanical in nature.

In terms of a general trend, the total adhesion force evolution with saturation is similar to the conceptual macroscopic representation through the 'suction stress characteristic curve' for sands as proposed by Lu \& Likos (2006) despite the difference in definition of the variables.

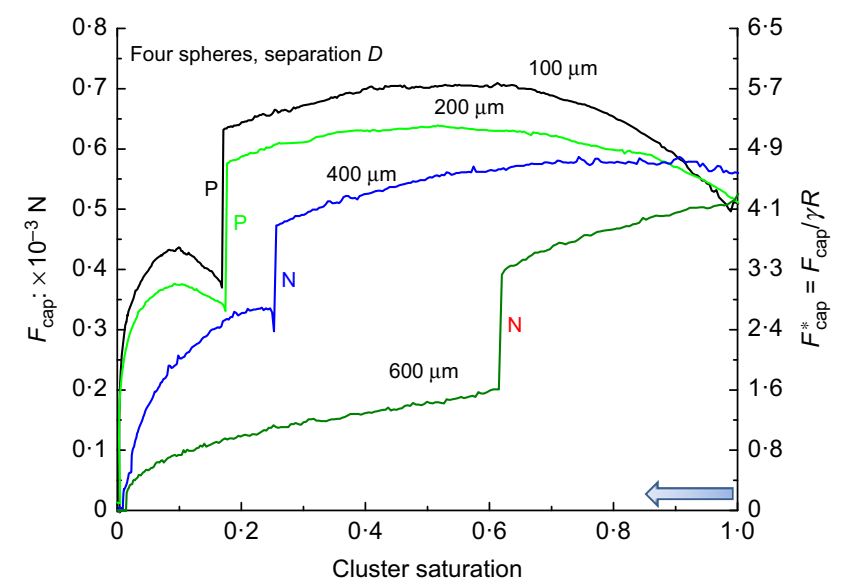

(a)

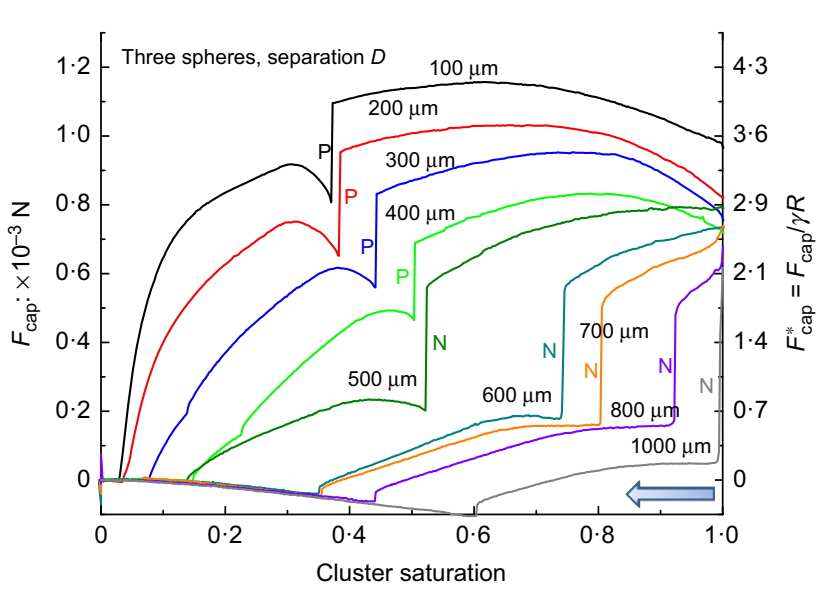

(b)

Fig. 4. Total adhesion force, $F_{\text {cap }}$, evolution during evaporation: (a) for a four-grain (3.5 mm dia.) cluster at different separations; (b) for a three-grain ( $8 \mathrm{~mm}$ dia.) cluster at different separations; $\mathbf{N}$, negative Gauss curvature point instability; $P$, positive Gauss curvature point instability 


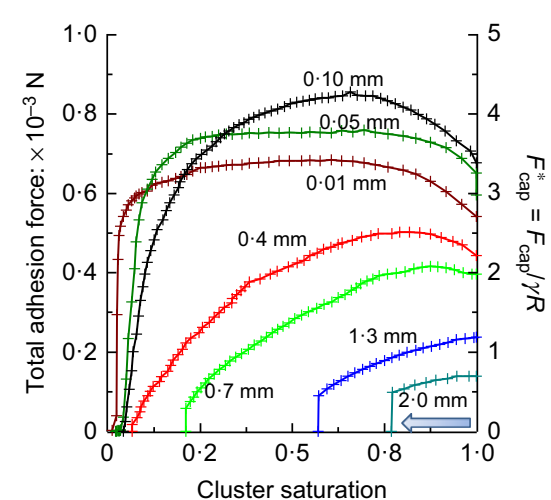

(a)

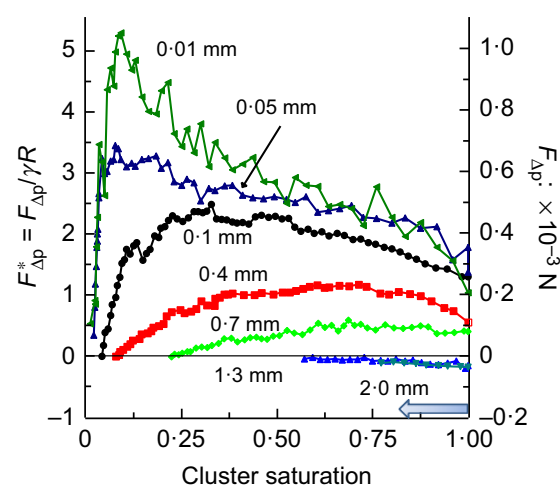

(b)

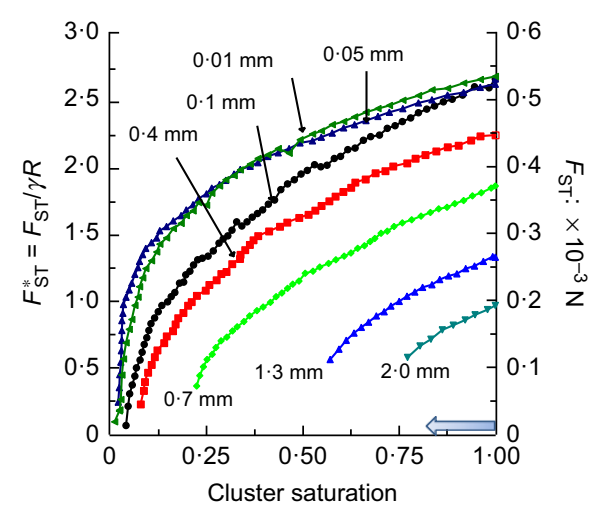

(c)

Fig. 5. (a) Total adhesion force, $F_{\text {cap }}$, evolution during evaporation for two-grain (8 mm dia.) pendular bridges at different separations. (b) Capillary pressure force, $F_{\Delta \mathrm{p}}$, component and (c) surface tension force, $F_{\mathrm{ST}}$, component of total intergranular (adhesive) force across two-grain capillary bridge (after Mielniczuk et al., 2015)

\section{GENERAL COMMENTS AND DISCUSSION}

In this study, different small wet clusters of grains have been examined and different patterns of the water body regression and the corresponding capillary force evolution during drying have been identified. Three different modes of air entry have been observed, perceived as local instabilities of the evolving water body, depending on the local GC of the water body surface (liquid-gas interface). The thin-sheet type instability at a bowl point and the finger instability at a saddle point develop for clusters of any number of grains, except for just two-grain systems, for which water wire pinching is the rupture mode. Water body evolution is briefly described using as an example a five-grain cluster, the biggest of all the clusters tested in this study, as it contains all observed instabilities and air-entry modes. Here, they are discussed for tight clusters merely to provide the context to the evolution of adhesive force.

For the tight clusters and at small separations between the rows $(0.1 \mathrm{~mm}$ and $0.3 \mathrm{~mm}$, e.g. Fig. 6) the first instability occurs through thinning of the water body from the front and back, at the central point (positive GC, bowl location). The thin water sheet subsequently develops an instability in the form of a circular hole, which expands into a curvilinear triangle, and then stabilises, creating a set of three- and two-grain bridges. Clearly, the two consecutive stable (equilibrium) configurations are characterised by a substantially different water mass distribution and its boundary. Also the resultant adhesion force values are different, producing a force jump. Each configurational instability implies a restructuring of suction, and the surface areas of liquid/solid contact, and configuration (length and inclination) of triple contact line. Further drying of the system leads to a subsequent evolution of three- and two-grain sub-system bridges, similar to individual three- and two-grain cluster bridges.

Clearly, the adhesion-force measured in the reported experiments is measured between the top row and the bottom row of the cluster. Hence, it is a particular measure, meaning it is not invariant of the force direction considered.
In all the cases considered here, it is an axial force acting along the axis of symmetry of the clusters tested. In an isotropic drying medium it is expected that intergranular adhesion-forces within a representative elementary volume (REV), would be uniformly distributed in all directions and could be converted into an isotropic tensor of adhesion back-stress. Performing a surface integration of the intergranular forces at an intersecting perimeter of the REV for a series of incrementally rotated vertically constrained clusters (see e.g. Drescher \& De Josselin de Jong, 1972) one would obtain such an averaged result of an adhesion back-stress for an equivalent continuum. That concept could not be applied here: the examined clusters tested cannot be considered as REVs, because of the limited number of grains.

Altogether, for all the tested clusters the adhesive force exhibits at least one jump at a constant cluster saturation, which constitutes a substantial fraction of the entire decrease of the force occurring during/due to evaporation. In Fig. 7(a) the summed (total) force jumps developed over the complete drying process per maximum force are plotted against separation distance $D$. For comparison, two-grain cluster data replicated from Mielniczuk et al. (2015) are included, explicitly, for $1 \cdot 3$, and $2 \mu \mathrm{m}$ separations. For the smaller separations for two grains, the jump is considered as originating from the onset of instability of Laplace pressure. It is noted that the intergranular force at the instant of the air entry clearly has a somewhat different meaning for each grain arrangement. For that reason in all clusters in which the measured force is between two top spheres and the bottom spheres (both five-grain and the four-grain clusters), the measured force is divided by 2 , to make it comparable to the systems with only one top sphere (three-grain and two-grain clusters).

The individual force jump amounts are roughly the same for all clusters. This is probably related to the two subsequent configurations of the water-gas interface, at which the water body can be in equilibrium. Interestingly, there seem to be
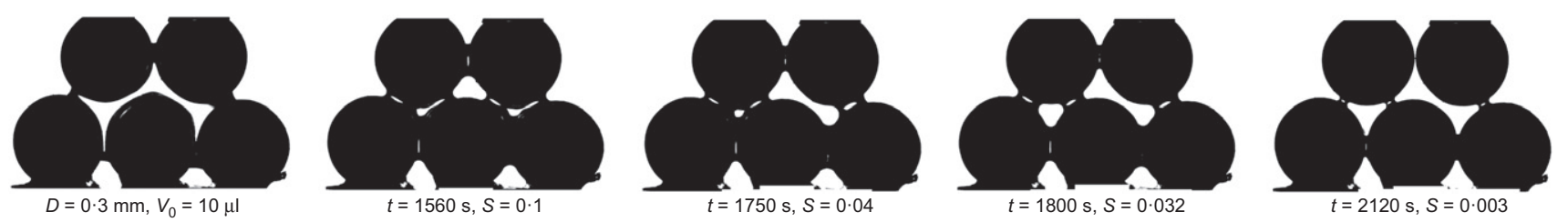

Fig. 6. Evolution of water body during evaporation (decrease of cluster saturation $S$ ) for five-grain tight clusters at $D=0 \cdot 3 \mathrm{~mm}$ 


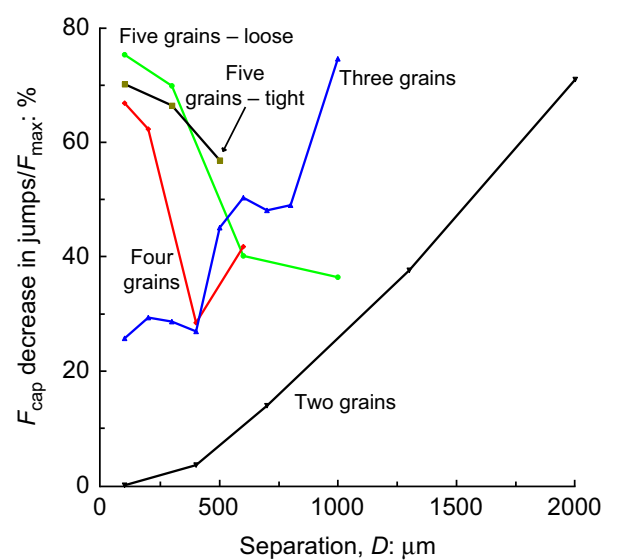

(a)

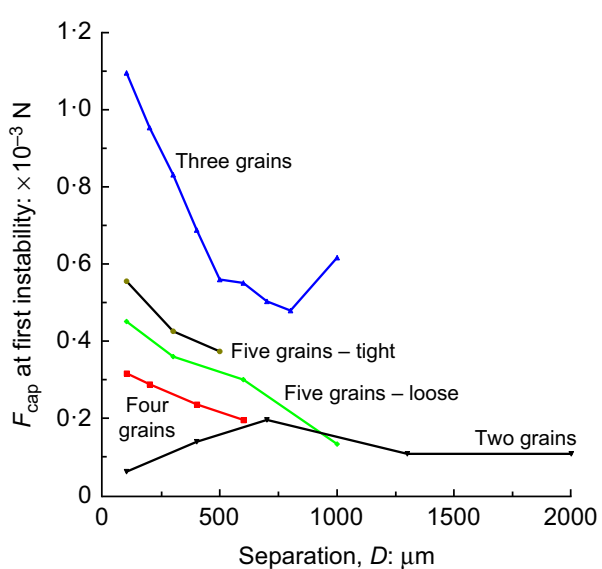

(b)

Fig. 7. (a) Total (added) force jumps per maximum force, $F_{\max }$, against grain separation; (b) intergranular (capillary) force, $F_{\text {cap }}$, at the moment of first air entry for different size clusters at different separations, $D$. It is higher for tight clusters than for the loose ones (at the same separations)

two tendencies developing: for the larger clusters, the jumps constitute a major part of the total force decrease at the smallest separations, while at large separations, the importance of jumps is smaller; for smaller clusters, an opposite tendency is seen, the biggest contribution of jumps is at the largest separations.

It is suggested that the difference of the energy expressed by way of half of the force-volume change product between the points of onset and end of the jump corresponds to the energy dissipated through the activation of water mass displacement by way of flow within the entire domain. The flow was not measured in the present experiments, but it is inferred from comparing the visible boundary changes between the points of jump onset and its end. Note that the flow is three-dimensional (3D) and includes invisible velocity components which are orthogonal to the plane seen in the images. An attempt to evaluate, at least approximately, the flow during the slow portion of the evaporation process for two spheres has been made by Yang et al. (2018). The recognition of the flow patterns arising during evaporation and those during instability episodes with the (fast) moving boundaries is of fundamental importance, as it is equivalent to the finding that drying of capillary bridges is a transient process and not a steady state.

The force jumps, are coincident with the fast motion of liquid/gas interfaces at points of both saddle-shaped and bowl-shaped external surface instability, discussed earlier and referred to as Haines jumps (Haines, 1930). Haines jumps have been extensively imaged and simulated numerically in recent years at meso-scale assemblies (Furuberg et al., 1996; Moebius \& Or, 2012; Berg et al., 2013). They appear to be random, both in terms of location and pressure, as well as in terms of timing, because all those depend on randomly distributed narrow pore throats. The evolution of the water body during both condensation into and evaporation from the granular clusters was thoroughly discussed by Melnikov et al. (2015) for two-, three- and more complex grain systems. They have adopted a number of geometrical criteria for liquid/liquid instabilities articulated earlier by Motealleh et al. (2013). Using Laplace-Young law they have calculated the pressure for smaller clusters, assuming particular contact angles. However, a hydromechanical pressure criterion for air entry (and progress) still awaits its formulation.

For larger assemblies, where pressure was monitored during a process of liquid volume injection into a non-wetting phase (Berg et al., 2013), it was observed that cooperative Haines jumps cascade through 10-20 pores per an unstable event, and consume about $64 \%$ of the energy of the medium drainage through dissipation. Saturation-driven dewatering tests, which more realistically reproduce the evaporation process, indicate that the resulting (negative) pressure is substantially lower than in the pressure-driven experiments (Cueto-Felgueroso \& Juanes, 2016). In large assemblies with a relatively high number of grains, the number of jumps per second is very high. In fine-grained soils, a drying front forms a band a few hundred grain diameters thick, where most of the desaturation process takes place (e.g. Armstrong et al., 2015). A useful example of such a result is reproduced in Fig. 8 after Berg et al. (2013). It shows that over a longer period of time the local pressure evolution time/volume in the presence of cooperative Haines jumps does not affect the global pressure-volume characteristics of the injection process, as seen in the inset. Thus, with a REV large enough (several hundred grains) and timescales long enough, as is the case in many geomechanics applications, Haines jumps are confined to a 'drying front' and become relatively inconspicuous saw-toothing of the averaged pressure, which appears to be a continuous and monotonic function of time; see also Måløy et al. (1992) and Georgiadis et al. (2013). Thus, it is expected that, upon homogenisation, an intergranular macro-scale force also becomes a continuous function of saturation, or water content (Lenormand et al., 1983; Berg et al., 2013; Helland et al., 2017).

However, from the point of view of the overall process of drying of soil, the first air entry, coincident with the first

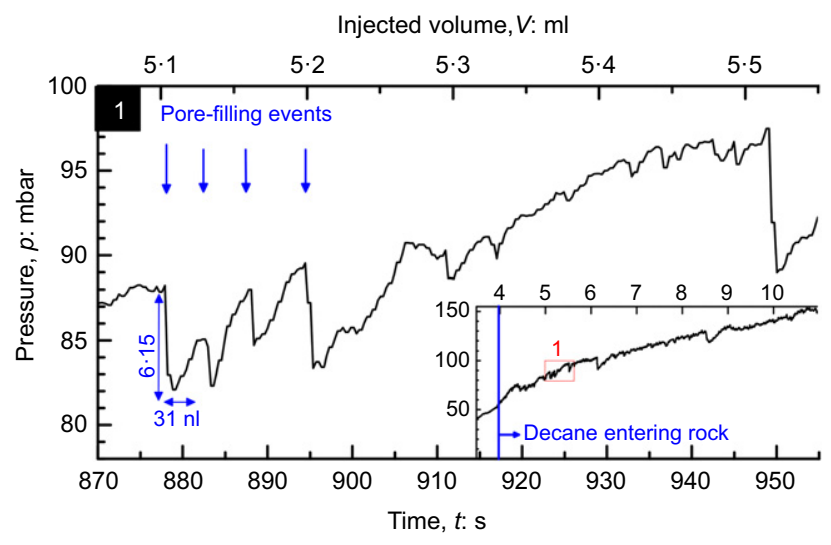

Fig. 8. Pore pressure evolution during non-wetting fluid (decane) injection into rock medium showing a distinct effect of Haines jumps at a small timescale, which become of less importance to long-term behaviour, as seen in the inset (reproduced from Berg et al. (2013)) 
Haines jump, is of fundamental importance as it marks the onset of desaturation. Currently, with the imaging techniques employed, it is not possible to provide a numerical value of the GC, and to possibly advance a hypothesis about a quantitative criterion for a critical air-entry pressure, or in fact, a criterion that would also include other variables, as those related to the surface tension resultant force. Further studies are needed to determine the quantitative and physical conditions for development of these instability modes and the depth of individual jumps.

As the focus of this study is on the intergranular force during drying up to rupture of a cluster, as a micro-scale manifestation of strength, it is of interest to assess the intergranular (capillary) force at the moment of first air entry. Fig. 7(b) shows the value of this force for different size clusters at different intergrain separations. As before, the force is per number of grains in the top row of the experiment. For comparison, the values of force at the onset of instability for a two-grain bridge have also been shown. It can be seen that the force value at the moment of air entry is smaller for larger separations, as expected. It is higher for tight clusters than for the loose ones (at the same separations). In general, as a reminder, for the tight clusters and smaller separations the air entry is through a bowl-point instability, while for the more loose clusters (larger separations) it is a saddleinstability. This is consistent with the general, although qualitative, observation that air entry occurs first at larger pores.

In addition, the morphology of the Haines jumps through the effect on the drying front depth and its displacement leads to saturation gradients and, implicitly, a rate dependency for the relative permeability (Xu et al., 2008; Berg et al., 2013). Fig. 7(a) shows the fraction of the maximum total force that is dissipated through the jumps. Haines jumps are also very important as possible total stress concentrators and potential triggers of drying cracking. Depth of the fingers is seen as critical for possible triggering of a drying crack (Holtzman \& Juanes, 2010; Hueckel et al., 2014).

On a separate issue of the representation of drying forces, remember that for two-grain bridges it is possible to assess the two components of the total intergranular force: the capillary pressure acting across the bridge gorge and the corresponding force, and the surface tension force acting along the bridge equatorial perimeter, Figs 5(b) and 5(c), Mielniczuk et al. (2015). Both components are attractive forces and are of the same order of magnitude. An exception from this occurs shortly before the moment of rupture for very slender bridges ( 1.3 and $2 \mathrm{~mm}$ tall in our case), in which the grains are repelled under a positive pressure, but attracted with a surface tension force. Equally important is the observation that both force components decrease to zero at the waning saturation, or at rupture. It is hence clear that ignoring the surface tension component from the consideration of cohesion eliminates 'half' of the force, and an important trend it represents of a consistent monotonic and accelerating decrease of the force. In fact, while the pressure resultant may evolve to be either negative or positive, the surface tension ensures that the total intergranular force remains attractive all the time. Yang et al. (2018) have calculated from the curvatures the local Laplace pressure along the height of the bridge, finding high-pressure gradients at the water/gas interface which evolve during evaporation. Thus, it is the present authors' belief that the proper representation of the cohesion of geomaterials during desaturation should be based on the adhesion force and should include both its components - that is suction force and surface tension perimeter force resultants.

Recent studies on three-grain and larger clusters reveal several important features of the similar mechanisms of evaporation presented here, including the two instability modes (Semprebon et al., 2016). However, they did not consider other states beyond equilibrium, or post-instability configurations and further evaporation. The behaviour during extension of three-grain bridges at constant volume was investigated by Wang et al. (2017). They analysed the process by considering a sequence of configurations in equilibrium, attributing the observed decrease of intergranular force to the evolution of the contact angle. The study did not address the states of the water body after the instabilities. It should be emphasised that no transient process analyses are known to date for the evaporation or re-morphing of capillary bridges.

On the macro-scale, measuring suction directly appears to be technically an easier task. On the micro-scale, to assess the adhesive force it is likewise important to follow the evolution of the specific surface area of the liquid/solid contact, over which the pressure (or suction) acts, see Fig. 9(a). The same pertains to the surface tension force and the length of the contact perimeter, Fig. 9(b). These two geometrical variables are not far from quadratic and linear functions, respectively, of the contact diameter, which tends to zero toward the end of the process. For the two-grain set, the contact diameter is seen to dramatically decrease in the terminal stages of drying, except for very tall bridges. Tall, pendular bridges rupture mechanically at early stages of drying, with almost constant contact diameter (pinning) over the entire process (see Mielniczuk et al., 2014a, 2015).

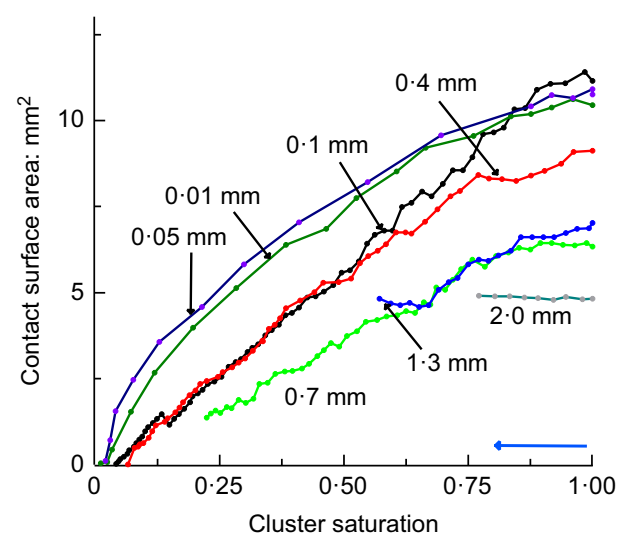

(a)

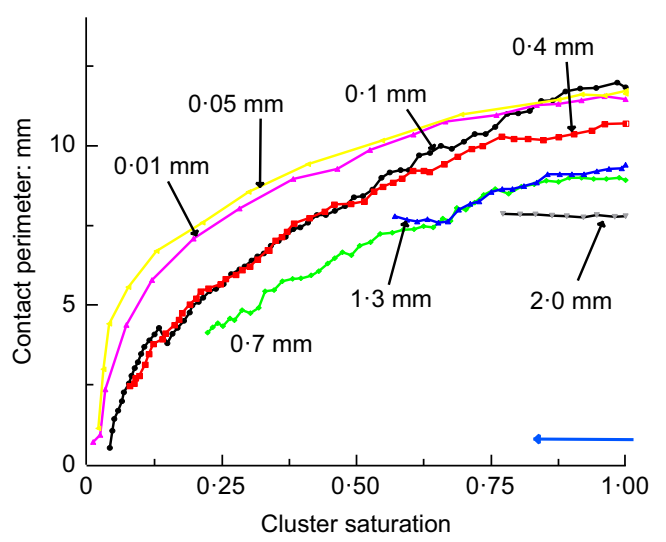

(b)

Fig. 9. (a) Liquid/solid contact surface area evolution during evaporation as a function of cluster saturations for two-grain clusters; (b) Triple phase contact line length evolution during evaporation as a function of cluster saturations for two-grain clusters 
Owing to the decay of the surface area over which suction is imparted on a grain, it is not surprising that the suction force goes to zero more dramatically than its surface tension counterpart, changing linearly with the contact diameter.

In macro-scale studies, especially in the area of contaminant transport, Hassanizadeh \& Gray (1993) introduced the specific surface area of the interfaces as an independent variable. However, as intergranular forces are not of interest in these applications, of all types of interfaces, only the wetting/non-wetting fluid interface area - that is water/air interface - was found of relevance and is included in the constitutive models (e.g. Joekar-Niasar et al., 2010). In practice, very few systematic studies of the role of the solid/liquid interface area are available, especially at the scale of physical phenomena involved, or at different levels of saturation.

Returning to the macro-scale, the collection of evidence in geotechnical engineering suggests that at very low water content the macro-scale cohesion decreases with the decrease in water content (Escario \& Sáez, 1986; Alonso et al., 1990; Gens, 2010). Such findings were explicitly confirmed by Richefeu et al. (2007) in direct shear tests for water content, $w<7 \%$, and by Soulié et al. (2006) for the uniaxial compression strength with water content below $10 \%$. Also, Lu et al. $(2007,2009)$ performed a series of extension tests on several varieties of sand to find that, for all of them, for a saturation less than $20 \%$ and/or for a water content below $5 \%$ (gravimetric), their tensile strength decreases with water content and saturation, from $1 \mathrm{kPa}$ at $10 \%$ saturation down to $0 \cdot 3 \mathrm{kPa}$ at $2 \cdot 5 \%$, whereas suction simultaneously increases substantially.

To evaluate the shear strength evolution during desaturation, calculations were performed of the capillary force maxima at the bridge rupture in extension for two-grain clusters by Rumpf (1962) and Schubert $(1975,1984)$ based on the intergranular force formulae (Fisher, 1926; Erle et al., 1971; Willett et al., 2000). A relationship between tensile strength and saturation degree has been obtained for all levels of saturation. Tensile strength and capillary pressure (after Schubert, 1984) exhibits a maximum strength during drying at a relatively high $(92 \%)$ saturation. The values are then decreasing with a decreasing saturation, both in funicular and pendular regimes, in particular below 4\%. This takes place despite a suggested dramatic increase of capillary pressure (at least theoretically, as no pressure data were reported below $25 \%$ of saturation). A similar trend in tensile strength is suggested by Lu et al. (2009) based on an empirical relationship between suction and saturation degree of van Genuchten (1980).

In summary, there is an apparent paradox in the widely reported extremely high values of macro-scale suction imposed on soil to yield very small water contents, and a decreasing, if not plummeting to zero, tensile and shear strength of the medium.

Two major factors that are revealed in micro-scale modelling of desaturation clarify, in the present authors' view, this apparent paradox: adhesion force is a resultant of two forces - namely, the suction force acting on the liquid/solid contact surface area, and the surface tension force acting along a triple contact perimeter. Both contact surface area and triple contact line decrease monotonically during the process and eventually tend to zero near the residual saturation (see Fig. 9). Hence, a description of unsaturated strength evolution of a granular microstructure by way of suction alone appears to be incomplete. In physical terms, it is not suction only, but also the eventually disappearing contact area across which it acts, and the surface tension force along the also eventually disappearing contact perimeter, that should be constitutive variables of interest that affect the adhesion, and hence the strength of desaturated granular matters, either directly, or through being a part of the adhesive force variable. Thus, phenomenological (macroscale) models that employ (like Laloui \& Nuth, 2009; Alonso et al., 2010) a product of suction and saturation, with the latter one that can be expressed by way of the liquid/solid contact radii, as a variable, offer most likely a chance to capture the mechanism evinced in the presented microstructural model. Alternative variables, such as interface surface area, concerning the water/air interface, do not directly play any role in the micro-structural model at hand, while the interface instability does trigger intergranular force jumps. This provides a motivation for including a contribution of both surface tension and water/air interface in the effective stress entity (e.g. Lu et al., 2010; Nikooee et al., 2013; Likos, 2014). However, any continuum effective stress defined per REV cannot be discussed at micro-scale, where all solid phase transmitted forces are carried by discrete intergranular forces.

It needs to be re-emphasised that all reported experiments were performed on non-deformable grain systems. From the numerous experiments with bridge extension it is clear that when the grains are displaced, the intergranular forces visibly change, but adhesion-forces at rupture do not. However, the deformation that would come from the compressibility of the grain in direct contact induced by capillary forces is likely to be immaterial.

Finally, it goes without saying that the models discussed, made of a low number of equal size, smooth, spherical glass grains that in the majority of cases are not in direct contact, and that are arranged in regular arrays, while intended to understand the mechanisms and trends of evolution of desaturating granular media, are not expected to duplicate quantitatively the behaviour of natural geological materials.

\section{CONCLUSIONS}

The experiments presented above lead to a number of findings, which can guide development of the constitutive modelling of variable saturation media, and can be summarised as follows.

(a) The reported micro-scale test results concerning the effect of evaporation on grain clusters of up to eight grains suggest that a proper representation of the cohesion of granular materials during desaturation should be based on the capillary force analysis and related to the total adhesion-force. Such a force should include both its components - namely, the pressure (suction) force and the surface tension perimeter force resultants. While this is well known in capillary fluid mechanics, the surface tension force and its evolution are often ignored in macroscopic unsaturated soil mechanics models. The pressure resultant force can be attractive or repulsive, and change its sense during evaporation, whereas the surface tension force is always attractive.

(b) Important factors of the evolution of the above identified components are two geometrical characteristics related to the micro-scale solid/liquid interface: the surface area of solid/liquid contact, over which pressure is to be integrated to yield the resultant force, and the length of the contact perimeter for the surface tension force. Both the surface area and the length of the contact perimeter decrease eventually to zero due to evaporation, either continuously or abruptly due to reconfiguration or rupture, see below. 
Both the surface area and the perimeter of contact are approximately (respectively, quadratic and linear) functions of the liquid/solid contact diameter. Notably, this is a different interface from the one discussed recently in some macro-scale models.

(c) The process of desaturation of discrete granular systems transitioning from capillary to funicular to pendular configurations is associated with a substantial evolution of the total adhesion (intergranular) force. The force is seen to rise to a maximum of two to three times higher value than the initial one, to subsequently decrease to zero, either continuously, when the water is evaporated, or abruptly, when bridge reconfigurations or rupture are involved.

(d) The decrease of the force associated with a gradual phase transition of water, is interrupted a number of times by discontinuities at constant saturations (Haines jumps) of the force value. These jumps, of an average of one-third of the current force value, are associated with unstable reconfigurations of the water interface with the air (so-called air entry) and are of three different types. The reconfigurations of the interface induce simultaneous displacements of water mass, necessarily implying internal water flows. The reconfigurations occur almost instantaneously and result in the formation of separate smaller bridges, which during subsequent drying, replicate the behaviour patterns of the small bridges, and/or isolated water film envelopes around isolated grains, that do not transmit any force.

(e) The discontinuities of the total force amount to between $95 \%$ of the maximum intergranular force and $20 \%$ of it over the process of desaturation.

( $f$ ) The Haines jumps, however, appear to be local events, when measured on meso- and macro-scale samples (as reported in the literature) and, over longer periods of time in terms of the associated adhesion-force, or pressure, do not seem to contribute substantially to the macro-scale adhesion-force evolution.

(g) The water body morphology changes, however, depend heavily on the fast water flows associated with Haines jumps and affect the development of the drying front depth (at meso-scale), displacement of water mass and its rate, which makes the entire process a transient process, far from, but tending eventually to, equilibrium configurations.

(h) The process of air entry (or first reconfigurations) is of critical importance as it creates initial conditions for drying cracking (Hueckel et al., 2014).

(i) A transition from understanding the mechanisms arising during evaporation of a desiccating granular medium at the scale of an assembly of a few grains and the associated water, to modelling the multi-physical behaviour of desaturation of a corresponding porous continuum, is not a trivial or straightforward undertaking. However, micro-scale models may offer valuable suggestions of variables of relevance, and their evolution and their limitations, together with the processes involved and methods to represent them mathematically in the process of up-scaling.

\section{ACKNOWLEDGEMENTS}

The authors acknowledge the partial support of US DOE grant DE-NE0008746 (T. Hueckel, B. Mielniczuk) and the partial support of MIST Laboratory (IRSNCNRS-University of Montpellier, B. Mielniczuk, M. S. El Youssoufi, T. Hueckel).

\section{REFERENCES}

Alonso, E., Gens, A. \& Josa, A. (1990). A constitutive model for partially saturated soils. Géotechnique 40, No. 3, 405-430, https://doi.org/10.1680/geot.1990.40.3.405.

Alonso, E. E., Pereira, J. M., Vaunat, J. \& Olivella, S. (2010). A microstructurally based effective stress for unsaturated soils. Géotechnique 60, No. 12, 913-925, https://doi.org/10.1680/ geot.8.P.002.

Armstrong, R. \& Berg, S. (2013). Interfacial velocities and capillary pressure gradients during Haines jumps. Phys. Rev. E 88, No. 4, 043010, https://doi.org/10.1103/PhysRevE.88.043010.

Armstrong, R. T., Evseev, N., Koroteev, D. \& Berg, S. (2015). Modeling the velocity field during Haines jumps in porous media. Adv. Wat. Resour. 77, 57-68.

Berg, S., Ott, H., Klapp, S. A., Schwing, A., Neiteler, R., Brussee, N., Makurat, A., Leu, L., Enzmann, F., Schwarz, J. O., Kersten, M., Irvinec, S. \& Stampanoni, M. (2013). Real-time 3D imaging of Haines jumps in porous media flow. PNAS 110, No. 10, 3755-3759.

Chang, C. S. \& Hicher, P. Y. (2009). Model for granular materials with surface energy forces. J. Aerospace Engng ASCE 22, No. 1, 43-54.

Cheng, D. C. H. (1968). The tensile strength of powders. Chem. Engng Sci. 23, No. 12, 1405-1420.

Cueto-Felgueroso, L. \& Juanes, R. (2016). A discrete-domain description of multiphase flow in porous media: rugged energy landscapes and the origin of hysteresis. Geophys. Res. Lett. 43, No. 4, 1615-1622, https://doi.org/10.1002/2015GL067015.

Deegan, R. D., Bakajin, O., Dupont, T. F., Huber, G., Nagel, S. R. \& Witten, T. A. (1997). Capillary flow as the cause of ring stains from dried liquid drops. Nature 389, No. 6653, 827-829.

Delage, P. (2013). Experimental techniques for unsaturated geomaterials. In Mechanics of unsaturated geomaterials (ed. L. Laloui), Ch. 4, pp. 89-112. Hoboken, NJ, USA: Wiley.

Drescher, A. \& De Josselin de Jong, G. (1972). Photoelastic verification of a mechanical model for the flow of a granular material. J. Mech. Phys. Solids 20, No. 5, 337-351.

Erle, M. A., Dyson, D. C. \& Morrow, N. R. (1971). Liquid bridges between cylinders, in a torus, and between spheres. AICHE J. 17, No. $1,115-121$.

Escario, V. \& Sáez, J. (1986). The shear-strength of partly saturated soils. Géotechnique 36, No. 3, 453-456, https://doi.org/10.1680/ geot.1986.36.3.453

Fisher, R. A. (1926). On the capillarity forces in an ideal soil; correction of formulae given by W. B. Haines. J. Agric. Sci. 16, No. 3, 492-505.

Fisher, L. R. \& Israelachvili, J. N. (1981). Experimental studies on the applicability of the Kelvin equation to highly curved concave menisci. J. Colloid Interf. Sci. 80, No. 2, 528-541.

Fredlund, D. G. \& Rahardjo, H. (1993). Soil mechanics for unsaturated soils. New York, NY, USA: John Wiley \& Sons.

Fredlund, D. G., Morgenstem, N. R. \& Widger, R. S. (1978). The shear strength of unsaturated soils. Can. Geotech. J. 15, No. 3, 313-321.

Furuberg, L., Måloy, K. J. \& Feder, J. (1996). Intermittent behavior in slow drainage. Phys. Rev. E 53, No. 1, 966-977.

Gens, A. (2010). Soil-environment interactions in geotechnical engineering. Géotechnique 60, No. 1, 3-74, https://doi.org/ 10.1680/geot.9.P.109.

Gens, A. \& Alonso, E. E. (1992). A framework for the behaviour of unsaturated expansive clays. Can. Geotech. J. 29, No. 6, 1013-1032.

Georgiadis, A., Berg, S., Maitland, G. \& Ott, H. (2013). Pore-scale microcomputed-tomography imaging: nonwetting-phase cluster-size distribution during drainage and imbibition. Phys. Rev. E 88, No. 33, 033002 .

Gras, J. P. (2011). Approche micromécanique de la capillarité dans les milieux granulaires: rétention d'eau et comportement mécanique. $\mathrm{PhD}$ thesis, Université Montpellier 2, Montpellier, France.

Gras, J. P., Delenne, J. Y. \& El Youssoufi, M. S. (2013). Study of capillary interaction between two grains: a new experimental device with suction control. Granular Matter 15, No. 1, 49-56.

Haines, W. B. (1930). Studies in the physical properties of soil. V. The hysteresis effect in capillary properties, and the modes of moisture distribution associated therewith. J. Agric. Sci. 20, No. $1,97-116$ 
Hassanizadeh, M. \& Gray, W. G. (1993). Thermodynamic basis of capillary pressure in porous media. Wat. Resour. Res. 29, No. 10, 3389-3405.

Helland, J. O., Friis, H. A., Jettestuen, E. \& Skjæveland, S. M. (2017). Footprints of spontaneous fluid redistribution on capillary pressure in porous rock. Geophys. Res. Lett. 44, No. 10, 4933-4943, https://doi.org/10.1002/2017GL073442.

Holtzman, R. \& Juanes, R. (2010). Crossover from fingering to fracturing in deformable disordered media. Phys. Rev. E 82, No. 4, 046305, Part 2.

Hotta, K., Takeda, K. \& Linoya, K. (1974). The capillary binding force of a liquid bridge. Powder Technol. 10, No. 4-5, 231-242.

Hueckel, T., Mielniczuk, B., El Youssoufi, M. S., Hu, L. B. \& Laloui, L. (2014). A three-scale cracking criterion for drying soils. Acta Geophys. 62, No. 5, 1049-1059, https://doi.org/ 10.2478/s11600-014-0214-9.

Israelachvili, J. N. (1992). Intermolecular and surface forces. London, UK: Academic Press.

Joekar-Niasar, V., Prodanović, M., Wildenschild, D. \& Hassanizadeh, S. M. (2010). Network model investigation of interfacial area, capillary pressure and saturation relationships in granular porous media. Wat. Resour. Res. 46, No. 6, W06526, https://doi.org/10.1029/2009WR008585.

Laloui, L. \& Nuth, M. (2009). On the use of the generalised effective stress in the constitutive modelling of unsaturated soils. Comput. Geotech. 36, No. 1-2, 20-23.

Lenormand, R., Zarcone, C. \& Sarr, A. (1983). Mechanisms of the displacement of one fluid by another in a network of capillary ducts. J. Fluid Mech. 135, 337-353.

Lian, G., Thornton, C. \& Adams, M. J. (1993). A theoretical study of the liquid bridge forces between two rigid spherical bodies. J. Colloid Interf. Sci. 161, No. 1, 138-147.

Likos, W. J. (2000). Total suction-moisture content characteristics for expansive soils. $\mathrm{PhD}$ dissertation, Colorado School of Mines, Golden, CO, USA.

Likos, W. J. (2014). Effective stress in unsaturated soil: accounting for surface tension and interfacial area. Vadose Zone J. 13, No. 5, vzj2013.05.0095.

Lu, N. \& Likos, W. J. (2004). Unsaturated soil mechanics. Hoboken, NJ, USA: John Wiley \& Sons.

Lu, N. \& Likos, W. J. (2006). Suction stress characteristic curve for unsaturated soil. J. Geotech. Geoenviron. Engng 132, No. 2, $131-142$.

Lu, N., Wu, B. \& Tan, C. P. (2007). Tensile strength characteristics of unsaturated sands. J. Geotech. Geoenviron. Engng 133, No. 2, 144-154.

Lu, N., Kim, T. H., Sture, S. \& Likos, W. J. (2009). Tensile strength of unsaturated sand. J. Engng Mech. 135, No. 12, 1410-1419, https://doi.org/10.1061/(ASCE)EM.1943-7889.0000054.

Lu, N., Godt, J. W. \& Wu, D. T. (2010). A closed-form equation for effective stress in unsaturated soil. Wat. Resour. Res. 46, No. 5, W05515, https://doi.org/10.1029/2009WR008646.

Luikov, A. V. (1966). Heat and mass transfer in capillary-porous bodies. Oxford, UK: Pergamon Press.

Måløy, K. J., Furuberg, L., Feder, J. \& Jøssang, T. (1992). Dynamics of slow drainage in porous media. Phys. Rev. Lett. 68, No. 14, 2161-2168.

Mason, G. \& Clark, W. C. (1965). Liquid bridges between spheres. Chem. Engng Sci. 20, No. 10, 859-866.

McQueen, I. S. \& Miller, R. F. (1974). Approximating soil moisture characteristics from limited data: empirical evidence and tentative model. Wat. Resour. Res. 10, No. 3, 521-527.

Melnikov, K., Mani, R., Wittel, F. K., Thielmann, M. \& Herrmann, H. J. (2015). Grain-scale modeling of arbitrary fluid saturation in random packings. Phys. Rev. E 92, No. 2, 022206, https://doi.org/10.1103/PhysRevE.92.022206.

Mielniczuk, B., Hueckel, T. \& El Youssoufi, M. S. (2014a). Evaporation-induced evolution of the capillary force between two grains. Granular Matter 16, No. 5, 815-828, https://doi.org/ 10.1007/s10035-014-0512-6.

Mielniczuk, B., El Youssoufi, M. S., Sabatier, L. \& Hueckel, T. (2014b). Rupture of an evaporating liquid bridge between two grains. Acta Geophysica 62, No. 5, 1087-1108, https://doi. org/10.2478/s11600-014-0225-6.

Mielniczuk, B., Hueckel, T. \& El Youssoufi, M. S. (2015). Laplace pressure evolution and four instabilities in evaporating two-grain liquid bridges. Powder Technol. 283, 137-151, https://doi.org/ 10.1016/j.powtec.2015.05.024.

Mitchell, J. K. (1976). Fundamentals of soil behavior. Hoboken, NJ, USA: John Wiley \& Sons.

Mitchell, J. K. \& Soga, K. (2005). Fundamentals of soil behavior, 3rd edn. Hoboken, NJ, USA: John Wiley \& Sons.

Moebius, F. \& Or, D. (2012). Interfacial jumps and pressure bursts during fluid displacement in interacting irregular capillaries. J. Colloid Interf. Sci. 377, No. 1, 406-415.

Mokni, N., Romero, E. \& Olivella, S. (2014). Chemohydro-mechanical behaviour of compacted Boom Clay: joint effects of osmotic and matric suctions. Géotechnique 64, No. 9, 681-693, https://doi.org/10.1680/geot.13.P.130.

Motealleh, S., Ashouripashaki, M., DiCarlo, D. \& Bryant, S. (2013). Unified model of drainage and imbibition in 3D fractionally wet porous media. Transp. Porous Media 99, No. 3, 581-611.

Murase, K., Mochida, T. \& Sugama, H. (2004). Experimental and numerical studies on liquid bridge formed among three spheres. Granular Matter 6, No. 2-3, 111-119.

Murase, K., Mochida, T., Sagawa, Y. \& Sugama, H. (2008). Estimation of the strength of a liquid bridge adhered to three spheres. Adv. Powder Technol. 19, No. 4, 349-367.

Newitt, D. M. \& Conway-Jones, J. M. (1958). A contribution to the theory and practice of granulation. Trans. Inst. Chem. Engrs 36, $422-441$.

Nikooee, E., Habibagahi, G., Hassanizadeh, S. M. \& Ghahramani, A. (2013). Effective stress in unsaturated soils: a thermodynamic approach based on the interfacial energy and hydromechanical coupling. Transp. Porous Media 96, No. 2, 369-396, https://doi.org/10.1007/s11242-012-0093-y.

Richefeu, V., El Youssoufi, M. S. \& Radjaï, F. (2007). Shear strength of unsaturated soils: experiments, DEM simulations, and micromechanical analysis. In Theoretical and numerical unsaturated soil mechanics (ed. T. Schanz), Springer Proceedings in Physics 113, pp. 83-91. Berlin/Heidelberg, Germany: Springer.

Romero, E., Della Vecchia, G. \& Jommi, C. (2011). An insight into the water retention properties of compacted clayey soils. Géotechnique 61, No. 4, 313-328, https://doi.org/10.1680/geot. 2011.61.4.313.

Rumpf, H. (1962). The strength of granules and agglomerates. In Agglomeration (ed. W. A. Knepper), pp. 379-418. New York, NY, USA: John Wiley \& Sons.

Schubert, H. (1975). Tensile strength of agglomerates. Powder Technol. 11, No. 2, 107-119.

Schubert, H. (1984). Capillary forces - modeling and application in particulate technology. Powder Technol. 37, No. 1, 105-116.

Semprebon, C., Scheel, M., Herminghaus, S., Seemann, R. \& Brinkmann, M. (2016). Liquid morphologies and capillary forces between three spherical beads. Phys. Rev. E 94, No. 1, 012907.

Soulié, F., Cherblanc, F., El Youssoufi, M. S. \& Saix, C. (2006). Influence of liquid bridges on the mechanical behaviour of polydisperse granular materials. Int. J. Numer. Analyt. Methods Geomech. 30, No. 3, 213-228.

Sun, D. A., Sheng, D. C., Cui, H. B. \& Sloan, S. W. (2007). A density-dependent elastoplastic hydro-mechanical model for unsaturated compacted soils. Int. J. Numer. Analyt. Meth. Geomech. 31, No. 11, 1257-1279.

Taylor, D. W. (1948). Fundamentals of soil mechanics. New York, NY, USA: Wiley.

Toussaint, R., Måløy, K. J., Méheust, Y., Løvoll, G. \& Jankov, M. (2012). Twophase flow: structure, upscaling, and consequences for macroscopic transport properties. Vadose Zone J. 11, No. 3, vzj2011.0123.

van Genuchten, M. T. (1980). A closed-form equation for predicting the hydraulic conductivity of unsaturated soils. Soil Sci. Soc. Am. J. 44, No. 5, 892-898.

Wang, J. P., Gallo, E., François, B., Gabrieli, F. \& Lambert, P. (2017). Capillary force and rupture of funicular liquid bridges between three spherical bodies. Powder Technol. 305, 89-98. https://doi.org/10.1016/j.powtec.2016.09.060.

Wheeler, S. J. \& Sivakumar, V. (1995). An elastoplastic critical state framework for unsaturated soil. Géotechnique 45, No. 1, 35-53, https://doi.org/10.1680/geot.1995.45.1.35. 
Willett, C. D., Adams, M. J., Johnson, S. A. \& Seville, J. P. K. (2000) Capillary bridges between two spherical bodies. Langmuir 16, No. 24, 9396-9405.

Xu, L., Davies, S., Schofield, A. B. \& Weitz, D. A. (2008). Dynamics of drying in 3D porous media. Phys. Rev. Lett. 101, No. 9, 094502 .
Yang, S., Mielniczuk, B., El Youssoufi, M. S. \& Hueckel, T. (2018). A note on evolution of pressure and flow within evaporating capillary bridge. Eur. Phys. J. E 41, No. 12, article 140, https://doi.org/10.1140/epje/i2018-11748-x.

Young, T. (1805). An essay on the cohesion of fluids. Phil. Trans. R. Soc. Lond. 95, 65-87. 